cerebral artery at base-line and after $30 \mathrm{~s}$ of breath holding. A breath-hold-index $(\mathrm{BHI})$ of $<0.69$ indicates impaired cerebrovascular reserve capacity.

Results IMT in the aortic arch was higher in patents versus controls, $1.3 \pm 0.3$ vs $1.1 \pm 0.2 \mathrm{~mm}(p=0.04)$, whereas no difference was found in the common carotid artery, 0.61 \pm 0.13 vs $0.55 \pm 0.10 \mathrm{~mm} \quad(p=0.2)$. BHI-values were lower in the SLE-group, $1.29 \pm 0.36$ vs $1.65 \pm 0.56 \quad(p=0.05)$, whereas both groups had signs of preserved cerebrovascular reserve capacity. Mean oxygen saturation peak was decreased in SLE patients versus controls, $79.5 \pm 7.8 \%$ vs $86.9 \pm 5.6 \% \quad(p=0.006)$. Endothelial function using EndoPAT did not differ, 0.72 \pm 0.40 vs $0.84 \pm 0.24,(p=0.3)$.

Conclusions This study indicates that microcirculatory vessel disease, as measured with EPOS and breath-hold index, could be present in SLE cases with atherosclerotic findings. However, the impaired microcirculation in SLE compared to population controls needs further validation in larger patient groups, also including non-atherosclerotic cases with SLE.

Funding Source(s): This work was supported by grants from the Swedish Rheumatism Association, the County Council of Östergötland, the Swedish Society of Medicine, the King Gustaf Vs 80 year anniversary foundation and the King Gustaf V and Queen Victorias Freemasons foundation.

\section{DYNAMIC DIFFUSE OPTICAL SPECTROSCOPY CAN DIAGNOSE AND QUANTIFY LUPUS ARTHRITIS}

${ }^{1}$ George Danias*, ${ }^{2}$ Youngwan Kim, ${ }^{2}$ Alessandro Marone, ${ }^{1}$ Kayla Neville, ${ }^{1}$ Tommy Chen, ${ }^{1}$ Andrea Frantz, ${ }^{1}$ Teja Kapoor, 'Laura Geraldino-Pardilla, ${ }^{2}$ Andreas Hielscher, ${ }^{2}$ Anca D Askanase. ${ }^{1}$ Columbia University Medical Center; ${ }^{2}$ Columbia University

\subsection{6/lupus-2019-Ism.97}

Background SLE arthritis is difficult to evaluate because of the sometimes-evanescent nature of the symptoms and limitations of physical exams and imaging studies. Dynamic diffuse optical spectroscopy (dDOS) can be used to assess changes in light absorption through tissues during transient venous occlusion. The optical signal reflects changes in blood perfusion and has diagnostic value in rheumatoid arthritis. The current study explored the use of dDOS in SLE arthritis.

Methods 12 SLE patients (ACR criteria) with active arthritis and 5 controls were evaluated. A dDOS sensor module was developed (figure. 1a). Hemodynamic effects were obtained by inflating a BP cuff to $40 \mathrm{mmHg} \times 60 \mathrm{~s}$. Light at 3 wavelengths (lambda $=530,655,940 \mathrm{~nm})$ was used to illuminate joints at 8 different points. Transmitted light intensities were measured with Si-photodetectors at 8 other positions (total $8 \times 8 \times 3=192$ signal traces). Swollen, tender and healthy joints were examined by the same assessor.

Results SLE patients and normal controls dDOS data were available for analysis from 66 and 24 proximal interphalangeal (PIP) joints, respectively (PIPs 2-4). Best results were obtained at $530 \mathrm{~nm}$ with cuff inflation at $40 \mathrm{mmHg}$. A representative measurement of 3 SLE arthritis and 3 normal joints is shown in figure $1 \mathrm{~b}$, highlighting differences in rise and plateau time. Given the pronounced effects at lambda $=530 \mathrm{~nm}$, we speculate that altered vessel physiology paired with already-increased blood pooling in the affected

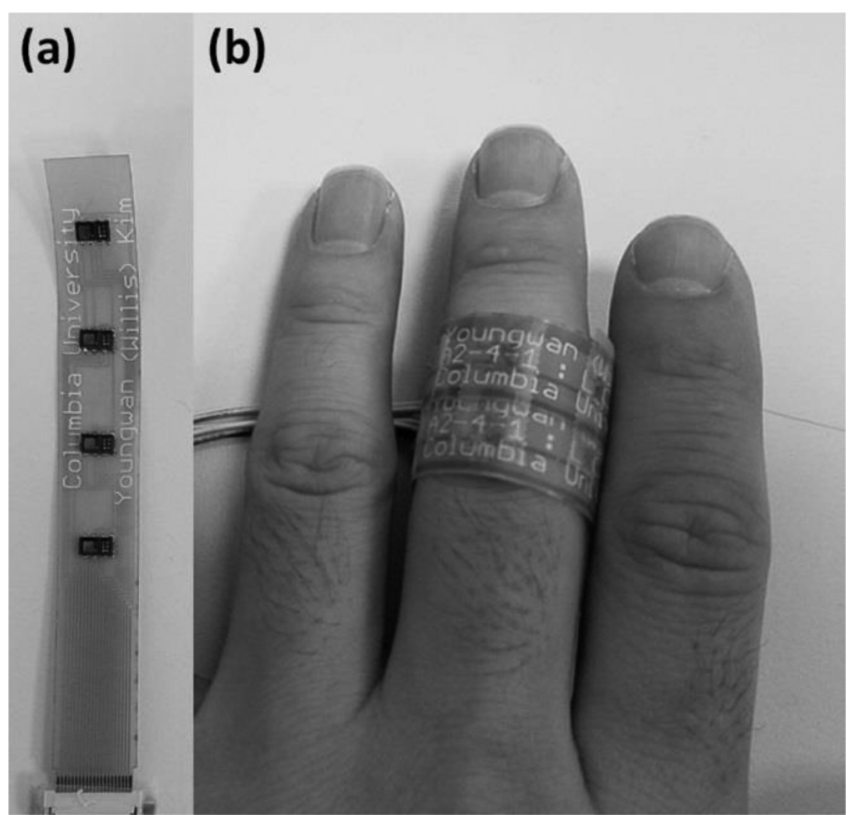

Abstract 97 Figure 1 a) Two dDOS sensor module bands (1a) wrapped around a PIP joint for measurement (1b). Each band contains 4 measurement heads (black dots). Each of the measurement heads includes 3 light-emitting diodes at three different wavelengths $(\lambda=530$ $\mathrm{nm}, 655 \mathrm{~nm}$, and $940 \mathrm{~nm}$, power $=2 \mathrm{~mW}$ ) and one Si-photodetector.

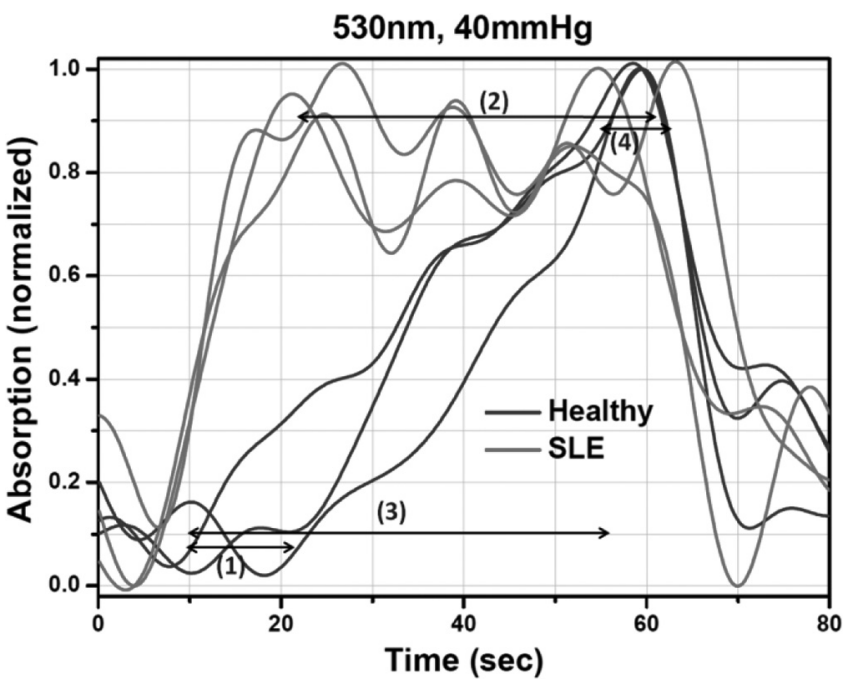

Abstract 97 Figure 1b Representative raw data for one healthy subject (blue) and one SLE arthritis subject (red). (1) and (2) are respectively the rise and the plateau times for a SLE patient, while (3) and (4) are respectively the rise and the plateau times for a healthy

inflamed joints resulted in quicker increase in light absorption (rise time) that is maintained longer (plateau time) compared to normal joints. The AUC for dDOS was consistent with excellent discrimination, $\mathrm{AUC}=0.8639$, sensitivity $=76.19$, specificity $=88.57$ (figure $1 \mathrm{c}$ ).

Conclusions dDOS can evaluate SLE arthritis with high sensitivity and specificity. Rise and plateau time of the optical traces correlate strongly with swollen and tender joint count. The advantages of dDOS are non-invasiveness, objectivity 
(a)

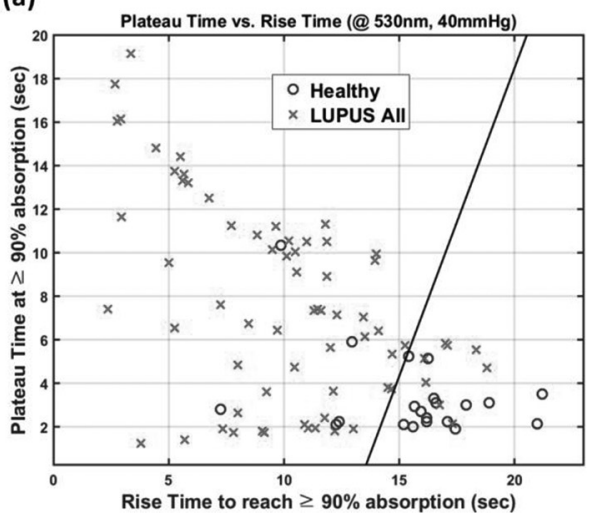

(b)

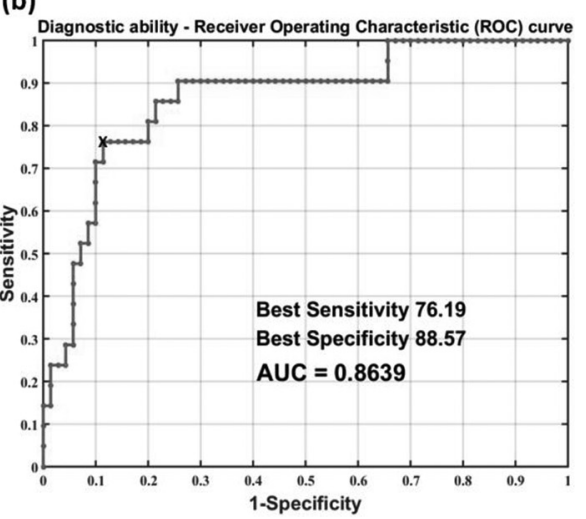

Abstract 97 Figure 1c Discriminant and ROC analysis which demonstrate the high specificity and sensitivity of dDOS when taking into account the rise time and the plateau time of the absorption signal (see Fig. 1b).

(eliminates inter-rater variability and operator dependency), low cost, and high speed of performance $(\sim 5 \mathrm{~min}$ per area of scanning) compared to US and MRI. dDOS has the potential to bring much-needed objectivity to the quantification of SLE arthritis.

Funding Source(s): N/A

\section{RESULTS OF A PHASE 2, DOUBLE-BLIND, RANDOMIZED, PLACEBO-CONTROLLED STUDY OF A REVERSIBLE B CELL INHIBITOR, XMAB ${ }^{\circledR} 5871$, IN SYSTEMIC LUPUS ERYTHEMATOSUS (SLE)}

${ }^{1}$ Joan T Merrill*, ${ }^{2}$ Joshua June, ${ }^{3}$ Fotios Koumpouras, ${ }^{4}$ Wambui Machua, ${ }^{5}$ Mohammed Faisal Khan, ${ }^{6}$ Anca D Askanase, ${ }^{7}$ Saira Z Sheikh, ${ }^{8}$ Arezou Khosroshahi, ${ }^{9}$ Paul Foster, ${ }^{9}$ Debra J Zack. 'Oklahoma Medical Research Foundation; ${ }^{2}$ Joshua June, DO; ${ }^{3}$ Yale Univeristy School of Medicine; ${ }^{4}$ Piedmont Atlanta Hospital; ${ }^{5}$ Arthritis and Rheumatology Center of Oklahoma, PLLC; ${ }^{6}$ Columbia University; ${ }^{7}$ University of North Carolina at Chapel Hill; ${ }^{8}$ Emory University; ${ }^{9}$ Xencor Inc

10.1136/lupus-2019-|sm.98
Background XmAb5871 is a humanized anti-CD19 antibody Fc-engineered for increased affinity to FcgammaRIIb. Co-ligation of CD19 and FcgammaRIIb inhibits B lineage cells key to lupus pathogenesis. This Phase 2 study in SLE was designed to minimize background medications and placebo responses to improve interpretation of a small trial in a complex, heterogenous disease.

Methods Patients were enrolled with active, non-organ threatening disease, and treated until improved with $160 \mathrm{mg}$ of IM Depo-Medrol. Immunosuppressive drugs were withdrawn except antimalarials or $10 \mathrm{mg} /$ day prednisone or equivalent before randomization to IV XmAb5871 (5 mg/kg) or placebo. Study treatments were given Q14 days until Day 225 or loss of improvement (LOI), defined as SLEDAI increase 4 points OR new BILAG A or B, with investigator-rated clinical significance. At LOI, patients resumed standard of care. The primary endpoint was the proportion of subjects without LOI by Day 225 in the efficacy evaluable group (those who completed Day 225 or discontinued due to LOI or a drug-related adverse event). Patients who withdrew for other reasons were excluded from this analysis.

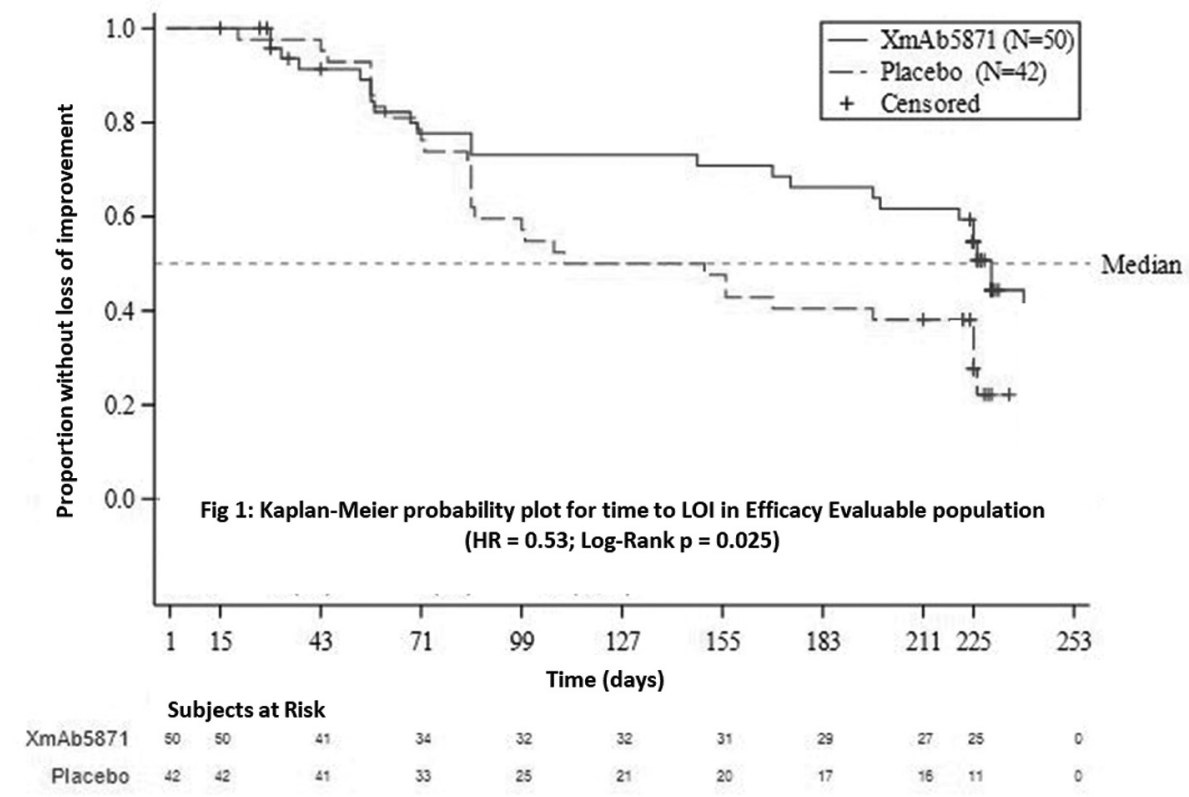

Abstract 98 Figure 1 Kaplan-Meier Probability Plot for Time to LOI in Efficacy Evaluable Populaion 